\title{
EXTENDING CONTINUOUS FUNCTIONS IN ZERO-DIMENSIONAL SPACES
}

\author{
NANCY M. WARREN
}

\begin{abstract}
Suppose that $X$ is a completely regular, zero-dimensional space and that a dense subset $S$ of $X$ is not $C^{*}$-embedded in $X$; does there then exist a two-valued function in $C^{*}(S)$ with no continuous extension to $X$ ? The answer is negative even if $X$ is a compact space. The question was raised by N. J. Fine and L. Gillman in Extension of continuous functions in $\beta N$, Bull. Amer. Math. Soc. 66 (1960), 376-381.
\end{abstract}

This paper answers a question raised by N. J. Fine and L. Gillman in [1]. Suppose that $X$ is a completely regular, zero-dimensional space and that a dense subset $S$ of $X$ is not $C^{*}$-embedded in $X$; does there then exist a two-valued function in $C^{*}(S)$ with no continuous extension to $X$ ? Theorem 1 establishes that the answer is negative.

I. First, I will give some background material, all of which can be found in [2].

All topological spaces are assumed to be completely regular.

The set of all bounded, continuous, real-valued functions on $X$ will be denoted by $C^{*}(X)$. A subspace $S$ of $X$ is $C^{*}$-embedded in $X$ iff every function in $C^{*}(S)$ can be extended to a function in $C^{*}(X)$. The Stone-Čech compactification of $X$ is denoted as $\beta X$; that is $\beta X$ is the compactification of $X$ in which $X$ is $C^{*}$-embedded.

A space $X$ is zero-dimensional if any two completely separated sets in $X$ are contained in complementary open-and-closed sets of $X$. A space $X$ is zero-dimensional if and only if $\beta X$ is zero-dimensional.

The space of countable ordinals with the order topology will be denoted by $W$.

II. Theorem 1. There exists a zero-dimensional space $X$ having a dense subset $S$ such that $S$ is not $C^{*}$-embedded in $X$, but every two-valued function in $C^{*}(S)$ has a continuous extension to $X$.

Proof. Let $I=[0,1]$ with the usual topology. For each $\alpha \in W$, select $I_{\alpha} \subset I$ such that $I_{\alpha}$ is dense in $I$ and $I_{\alpha} \cap I_{\beta}=\phi$ if $\alpha \neq \beta$, and such that $\bigcup_{a \in W} I_{\alpha}=I$.

Received by the editors March 1, 1974 and, in revised form, June 20, 1974. AMS (MOS) subject classifications (1970). Primary 54C45; Secondary 54C20, $54 \mathrm{G} 20$.

Key words and phrases. Zero-dimensional space, $C^{*}$-embedded. 
Let $S_{a}=\left\{\langle x, a\rangle: x \in \bigcup_{\beta \leq a} I_{\beta}\right\}$ and $S=\bigcup_{a \in W} S_{a^{*}}$ Then $S \subset I \times W$. Topologize $S$ using the relative topology from $I \times W$.

Note that the collection of all neighborhoods of $\langle x, \alpha\rangle$ of the form $\{\langle r, y): y<r<z$ and $\delta<y \leq a\}$ where $y<x<z$ and $y$ and $z$ belong to $\bigcup_{\beta>a} I_{\beta}$ is a basis of open-closed neighborhoods of $\langle x, \alpha\rangle$ since $\bigcup_{\beta>\alpha} I_{\beta}$ is dense in $I$.

Let $X=S \cup\{2\}$. We define a topology on $X$ as follows. $S$ will be an open subspace of $X$. A neighborhood of 2 is any set $U$ containing 2 such that $2 \in U$ and there is a $\beta \in W$ such that $\{\langle x, \alpha\rangle: \alpha>\beta\} \subset U$.

Since every neighborhood of 2 intersects $X, S$ is dense in $X$. Also, $S$ is completely regular since $S \subset I \times W$ where both $I$ and $W$ are completely regular.

A consequence of the following proof that $X$ is zero-dimensional is that $X$ is normal. So $X$ is, clearly, completely regular.

To show that $X$ is zero-dimensional, I will show that any two disjoint closed sets in $X$ are contained in complementary open-closed sets. First, consider the case where $A$ and $B$ are disjoint closed sets in $X$ such that $A \cup B \subset C=\bigcup_{a \leq y} S_{\alpha}$, for some $\gamma \in W$. Consider $\alpha_{0} \leq \gamma$. For each point $\left\langle x, a_{0}\right\rangle \in S_{a_{0}}$ pick $U(x)$ a basic open-closed neighborhood of $\left\langle x, a_{0}\right\rangle$ such that either $U(x) \cap A$ or $U(x) \cap B$ is empty. Identifying $S_{a_{0}}$ with $I$ $\bigcup_{\beta>a_{0}} I_{\beta}, S_{\alpha_{0}}$ is second countable, so a countable collection $\left\{U(x)_{n}\right\}_{n \in N}$ covers $S_{a_{0}}$. Now, since $\gamma \in W$ there is a countable collection, say $\left\{V_{n}\right\}_{n \in N^{\prime}}$ of open-closed sets, covering $C$ with the property that for each $n$, either $V_{n} \cap A$ or $V_{n} \cap B$ is empty. Define $W_{n}=V_{n}-\bigcup_{i<n} V_{i}$. Then $\left\{W_{n}\right\}_{n \in N}$ is a collection of disjoint open-closed sets which covers $C$ and either $W_{n} \cap A$ or $W_{n} \cap B$ is empty. Let $0=\bigcup\left\{W_{k}: W_{k} \cap A=\phi\right\}$; then $C-0=\bigcup\left\{W_{k}: W_{k} \cap\right.$ $A \neq \phi\}$. So 0 and $C-0$ are complementary open-closed sets in $C$ and $A C$ $C-0$ and $B \subset 0$. Since $X-C$ is open and closed in $X, 0 \cup X-C$ and $C-0$ are complementary open-closed sets in $X$.

Now, suppose $A$ and $B$ are disjoint closed sets in $X$ and $2 \in A$. Then there exists a $\beta \in W$ such that $B \subset D=\bigcup_{\alpha \leq \beta} s_{\alpha}$. Since $D$ is closed in $X$, $A \cap D$ is closed in $X$. By the above argument there exist complementary open-closed sets $H$ and $K$ in $D$ such that $B \subset H$ and $A \cap D \subset K$. Then $H$ and $K \cup X-D$ are complementary open-closed sets in $X$ such that $B \subset H$ and $A \subset H \cup X-D$. So $X$ is zero-dimensional.

To show that $S$ is not $C^{*}$-embedded in $X$, define $F: S \rightarrow I$ by $F(\langle x, a\rangle)$ $=x$. Obviously $F$ is continuous.

However, $F$ cannot be extended continuously to 2 , since $F$ assumes all values in every neighborhood of 2 .

Every two-valued continuous function on $S$ can be extended continuously to $X$. Let $f$ be a two-valued continuous function on $S$ with range $\{0,1\}$. For 
each $x \in I$, there exists an $\alpha_{x} \in W$ such that $f$ is constant on $\{\langle x, \beta\rangle: \beta \geq$ $\left.\alpha_{x}\right\}$, since for fixed $x$, the set of all points $\langle x, \alpha\rangle \in S$ is homeomorphic to $W$.

Now, for each $x \in I, x \neq 0,1$, there exists an integer $N_{x}$ such that $f$ is constant on

$$
U_{x}=\left\{\langle y, a\rangle: x-1 / N_{x}<y<x+1 / N_{x}, a>\alpha_{x}\right\} .
$$

If not, then for every integer $n$, there is a point $\left\langle y_{n}, \alpha_{n}\right\rangle$ such that $x-1 / n$ $<y_{n}<x+1 / n$ and $\alpha_{n}>\alpha_{x}$ and $f\left(\left\langle y_{n}, a_{n}\right\rangle\right) \neq f\left(\left\langle x, \alpha_{x}\right\rangle\right)$. But $x$ is the limit of $\left\{y_{n}\right\}$ and some $\alpha^{\prime} \in W$ is the limit of $\left\{a_{n}\right\}$, so by the continuity of $f$, $f\left(\left\langle x, \alpha^{\prime}\right\rangle\right) \neq f\left(\left\langle x, a_{x}\right\rangle\right)$ which is a contradiction since $\alpha^{\prime}>a_{x}$. Similar arguments establish the existence of $U_{0}$ and $U_{1}$.

For each $U_{x}$, consider $U_{x}^{\prime}=\left(x-1 / N_{x}, x+1 / N_{x}\right) \subset I$. The collection $\left\{U_{x}^{\prime}: x \in I\right\}$ is an open cover of $I$. Pick a finite subcover $\left\{U_{x_{i}}^{\prime}\right\}_{i=1}^{k}$. $B=\bigcup_{\beta>a_{x_{1}}} s_{\beta}$.

Extend $f$ to $f^{\prime}: X \rightarrow\{0,1\}$ by defining $f^{\prime}(2)=f(B)$. Clearly $f^{\prime}$ is continuous at 2 since $B \cup\{2\}$ is a neighborhood of 2 .

III. Corollary. There exists a zero-dimensional compact space which satisfies Theorem 1 .

Proof. Since $X$ is zero-dimensional, $\beta X$ is zero-dimensional and $S$ is dense in $\beta X$. Since $F \in C^{*}(S)$ cannot be extended to $X, F$ cannot be extended to $\beta X$. But every two-valued function in $C^{*}(S)$ extends to $X$ and hence to $\beta X$. So $\beta X$ is a compact zero-dimensional space which satisfies Theorem 1.

\section{REFERENCES}

1. N. J. Fine and L. Gillman, Extension of continuous functions in $\beta N$, Bull. Amer. Math. Soc. 66 (1960), 376-381. MR 23 \#A619.

2. L. Gillman and M. Jerison, Rings of continuous functions, University Ser. in Higher Math., Van Nostrand, Princeton, N. J., 1960. MR 22 \#6994.

DEPARTMENT OF MATHEMATICS, METROPOLITAN STATE COLLEGE, DENVER, COLORADO 80222 